\title{
Uniformization and anti-uniformization properties of ladder systems
}

\author{
by \\ Zoltán Balogh, Todd Eisworth (Cedar Falls, IA), \\ Gary Gruenhage (Auburn, AL), Oleg Pavlov (Towson, MD) \\ and Paul Szeptycki (Toronto)
}

\begin{abstract}
Natural weakenings of uniformizability of a ladder system on $\omega_{1}$ are considered. It is shown that even assuming $\mathrm{CH}$ all the properties may be distinct in a strong sense. In addition, these properties are studied in conjunction with other properties inconsistent with full uniformizability, which we call anti-uniformization properties. The most important conjunction considered is the uniformization property we call countable metacompactness and the anti-uniformization property we call thinness. The existence of a thin, countably metacompact ladder system is used to construct interesting topological spaces: a countably paracompact and nonnormal subspace of $\omega_{1}^{2}$, and a countably paracompact, locally compact screenable space which is not paracompact. Whether the existence of a thin, countably metacompact ladder system is consistent is left open. Finally, the relation between the properties introduced and other well known properties of ladder systems, such as $\boldsymbol{p}$, is considered.
\end{abstract}

1. Introduction. Let $S$ denote a stationary subset of limit ordinals of $\omega_{1}$. A ladder system on $S$ is a sequence $\left\{L_{\alpha}: \alpha \in S\right\}$ such that each $L_{\alpha}$ is an unbounded subset of $\alpha$ of order type $\omega$.

A ladder system is uniformizable if for each sequence $\left\langle f_{\alpha}: \alpha \in S\right\rangle$ of functions $f_{\alpha}: L_{\alpha} \rightarrow \omega$ there is an $F: \omega_{1} \rightarrow \omega$ such that $F\left\lceil L_{\alpha}={ }^{*} f_{\alpha}\right.$ for each $\alpha \in S$, i.e., for each $\alpha \in S$,

$$
\left\{\beta \in L_{\alpha}: F(\beta) \neq f_{\alpha}(\beta)\right\} \text { is finite. }
$$

2000 Mathematics Subject Classification: Primary 03E05, 54D15; Secondary 03E35, $03 \mathrm{E} 75$.

Key words and phrases: ladder systems, uniformizable, normal, countably paracompact, screenable.

Sections 5 and 6, as well as Theorem 14 and Corollary 15, represent joint work of the first and third authors conducted at the University of Wisconsin in the Fall of 1989.

The third author acknowledges support from NSF grant DMS-0072269.

The fifth author acknowledges support from NSERC grant 238944. 
We now formulate natural weakenings of uniformizable denoted, for each $n \in \omega$, by $\mathcal{P}_{n}:$ A ladder system is said to satisfy $\mathcal{P}_{n}$ if for each $f: S \rightarrow \omega$ there is an $F: \omega_{1} \rightarrow[\omega]^{n+1}$ such that for each $\alpha \in S$,

(a) $F \nmid L_{\alpha}$ is eventually constant with value $s_{\alpha}$,

(b) $f(\alpha) \in s_{\alpha}$.

Note that $\mathcal{P}_{0}$ is equivalent to the version of uniformizable obtained by considering only sequences of constant functions $f_{\alpha}$.

We will say that a ladder system satisfies $\mathcal{P}_{<\omega}$ if for each $f: S \rightarrow \omega$ there is an $F: \omega_{1} \rightarrow[\omega]^{<\omega}$ satisfying (a) and (b) above.

If we drop the requirement that the restrictions $F\left\lceil L_{\alpha}\right.$ are eventually constant we obtain uniformization properties that we denote by $\mathcal{M}_{n}$ and $\mathcal{M}_{<\omega}$. For example, a ladder system is said to satisfy $\mathcal{M}_{<\omega}$ if for each $f: S \rightarrow \omega$ there is an $F: \omega_{1} \rightarrow[\omega]^{<\omega}$ such that for each $\alpha \in S, f(\alpha) \in F(\beta)$ for all but finitely many $\beta \in L_{\alpha}$.

Most of these uniformization properties can be characterized in terms of properties of a certain topological space naturally associated to any ladder system. If $L$ is a ladder system, let $X_{L}$ denote the topological space $\omega_{1} \times$ $\{0\} \cup S \times\{1\}$ where every point $(\alpha, 0)$ is isolated and for each $\alpha \in S$, a basic neighborhood of $(\alpha, 1)$ consists of $\{(\alpha, 1)\}$ along with a cofinite subset of $L_{\alpha} \times\{0\}$. Such a space is always first countable and locally compact. The stationarity of $S$ implies that it is not collectionwise Hausdorff.

Spaces $X_{L}$ have been considered by many to construct examples of normal not collectionwise Hausdorff spaces (see [11] and [2]). It is folklore that a ladder system $L$ satisfies $\mathcal{P}_{0}$ if and only if $X_{L}$ is normal. The property $\mathcal{M}_{<\omega}$ is characterized by $X_{L}$ being countably metacompact. For this reason, we will say that a ladder system $L$ is countably metacompact if it satisfies $\mathcal{M}_{<\omega}$.

Claim 1. Let $L$ be a ladder system on a stationary set $S$. Then $X_{L}$ is countably metacompact if and only if $L$ satisfies $\mathcal{M}_{<\omega}$.

Proof. $X_{L}$ is countably metacompact if and only if each partition of $S$ into countably many sets has an open expansion which is point-finite on the isolated points. Suppose $f: S \rightarrow \omega$. By countable metacompactness, fix a point-finite open expansion $\left\{U_{n}: n \in \omega\right\}$. Let $F(\beta)=\left\{n: \beta \in U_{n}\right\}$. Clearly $F: \omega_{1} \rightarrow[\omega]^{<\aleph_{0}}$ is as required. Conversely, given a partition and a corresponding function $f$ fix the $F$ given by $\mathcal{M}_{<\omega}$. Define $U_{n}=f^{-1}(n) \cup$ $\left\{\beta \in \omega_{1}: n \in F(\beta)\right\}$. It is straightforward to check that $\left\{U_{n}: n \in \omega\right\}$ is the required point-finite open expansion.

The additional conclusion in $\mathcal{P}_{<\omega}$ that $F\left\lceil L_{\alpha}\right.$ is eventually constant corresponds to the open expansion being locally finite, hence $\mathcal{P}_{<\omega}$ implies that $X_{L}$ is countably paracompact. We do not know whether the two properties are equivalent. 


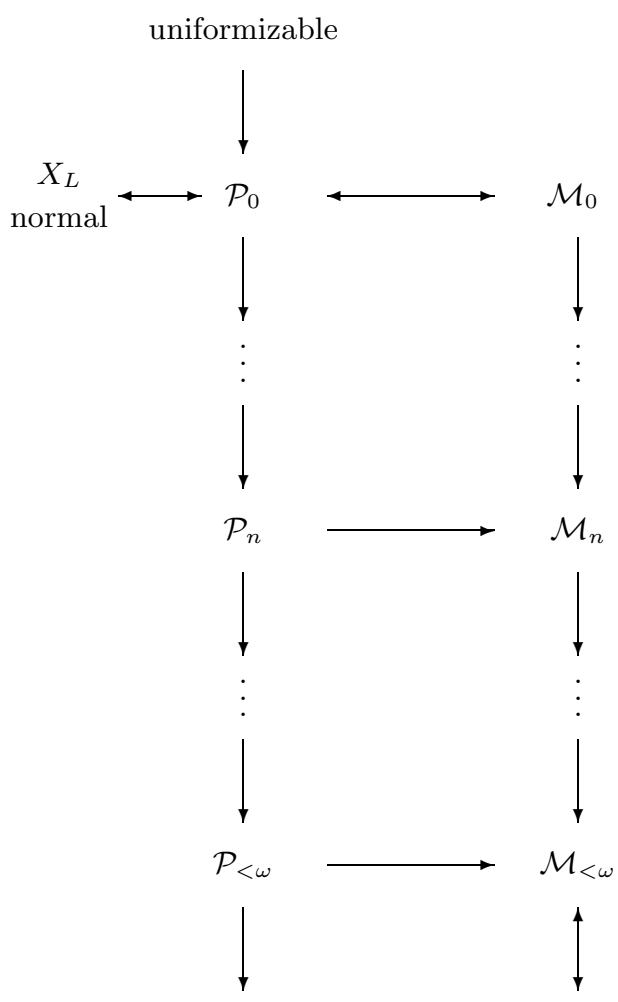

$X_{L}$ ctbly parac. $\longrightarrow X_{L}$ ctbly metac.

Fig. 1

QUESTION 1. If $X_{L}$ is countably paracompact, does L satisfy $\mathcal{P}_{<\omega}$ ?

The diagram of known ZFC implications between all the properties is given in Figure 1.

It should be remarked that $\mathrm{MA}\left(\omega_{1}\right)$ implies that every ladder system is uniformizable and that $2^{\aleph_{0}}<2^{\aleph_{1}}$ implies that no ladder system is uniformizable (see [6]). However, there is a ladder system on $\omega_{1}$ with the property $\mathcal{M}_{<\omega}$. In fact, any ladder system $L$ with the property that the $n$th element of each ladder $L_{\alpha}$ is of the form $\beta+n$ with $\beta$ a limit ordinal has property $\mathcal{M}_{<\omega}$. Indeed, let the function $F$ on $\omega_{1}$ be defined by $F(\alpha)=\{0,1, \ldots, n\}$, where $\alpha$ is of the form $\beta+n$ for some limit ordinal $\beta$. Then $F$ uniformizes every function $f$ in the sense of $\mathcal{M}_{<\omega}$. However, it is not hard to see that $\mathrm{V}=\mathrm{L}$ implies that no ladder system on a stationary subset of $\omega_{1}$ can satisfy $\mathcal{P}_{<\omega}$ (see the remark following the proof of Theorem 24).

In Section 2 of this paper we prove that for each $n \in \omega$ it is consistent with $\mathrm{CH}$ that for every stationary set $S$ and every ladder $L$ on $S, L$ satisfies $\mathcal{P}_{n+1}$ but does not satisfy $\mathcal{M}_{n}$. Thus, there are no other ZFC implications between the properties $\mathcal{P}_{n}$ and $\mathcal{M}_{m}$ for any $m, n<\omega$. Moreover, by taking 
$n=0$, we find that it is consistent with $\mathrm{CH}$ that every ladder system space $X_{L}$ is countably paracompact (in a strong sense) but not normal.

This leaves a few questions open, including the following:

Question 2. Is it consistent that all ladder systems satisfy $\mathcal{P}_{<\omega}$ but not $\mathcal{P}_{n}$ for any $n$ ?

The next set of properties of ladder systems we will consider are in some sense anti-uniformization properties. The following is the strongest of these. A ladder system with this property will also be called thin.

$\left(G_{1}\right) \quad$ For each $f: \omega_{1} \rightarrow \omega$ the set $\left\{\alpha \in S:\left|f^{\prime \prime} L_{\alpha}\right|=\aleph_{0}\right\}$ is nonstationary.

By strengthening what $f$ is allowed to do on a nonstationary set, we obtain the following weakenings of $G_{1}$ :

$\left(G_{2}\right)$ For each $f: \omega_{1} \rightarrow \omega$ the set $\left\{\alpha \in S: f\left\lceil L_{\alpha}\right.\right.$ is finite-to-one $\}$ is nonstationary.

$\left(G_{3}\right) \quad$ For each $f: \omega_{1} \rightarrow \omega$ the set $\left\{\alpha \in S: f\left\lceil L_{\alpha}\right.\right.$ is eventually one-to-one $\}$ is nonstationary.

By instead demanding that every $f$ fails to have certain properties on a stationary set, we obtain even weaker properties:

$\left(H_{1}\right) \quad$ For each $f: \omega_{1} \rightarrow \omega$ the set $\left\{\alpha \in S:\left|f^{\prime \prime} L_{\alpha}\right|<\aleph_{0}\right\}$ is stationary.

$\left(H_{2}\right) \quad$ For each $f: \omega_{1} \rightarrow \omega$ the set $\left\{\alpha \in S: f\left\lceil L_{\alpha}\right.\right.$ is not finite-to-one $\}$ is stationary.

$\left(H_{3}\right) \quad$ For each $f: \omega_{1} \rightarrow \omega$ the set $\left\{\alpha \in S: f\left\lceil L_{\alpha}\right.\right.$ is not eventually one-to-one $\}$ is stationary.

So, from the definitions we get the diagram of implications in Figure 2.

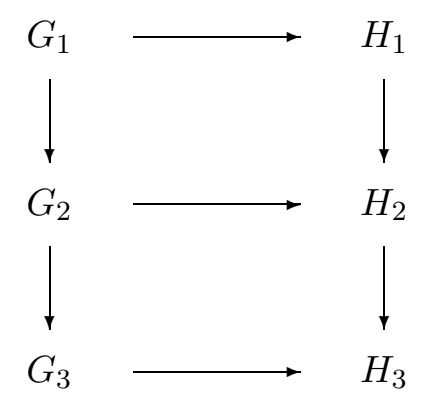

Fig. 2

Note that if a ladder system $L$ is uniformizable, then it fails to satisfy $H_{3}$. Indeed, any $F$ uniformizing a sequence of one-to-one functions $\left\langle f_{\alpha}\right\rangle$ will witness the failure of $\mathrm{H}_{3}$. However, some of the weaker versions of uniformizable can be consistent with some of the anti-uniformization properties. In [11], Shelah proved it consistent that there is a ladder system $L$ on a stationary 
set such that $X_{L}$ is normal and the closed discrete set of nonisolated points is not a $G_{\delta}$-set. Using our terminology, Shelah's ladder system satisfies $\mathcal{P}_{0}$ and $H_{2}$. Burke and Balogh [2] proved it consistent that there is a ladder system defined on a club subset of $\omega_{1}$ satisfying $\mathcal{M}_{<\omega}$ and $H_{2}$.

The most interesting open question is whether it is consistent that there is a ladder system satisfying $G_{1}$ and $\mathcal{M}_{<\omega}$. Much of this paper is devoted to these properties and henceforth we adopt the more descriptive names thin and countably metacompact.

In Section 3 we give two proofs establishing the consistency of the existence of a thin ladder system. We also consider the existence of thin ladders on stationary subsets of cardinals greater than $\omega_{1}$.

In Section 4 we consider the question whether every countably paracompact subspace of $\omega_{1}^{2}$ is normal. With a ladder $L$ on a stationary set $S$, we associate a nonnormal subspace $Z_{L} \subseteq \omega_{1}^{2}$. The main result of that section is that $Z_{L}$ is countably paracompact if and only if $L$ is thin and countably metacompact. We also consider another closely related construction of a subspace of $\omega_{1}^{2}$.

Section 5 is devoted to the following open problem: Is there (consistently) a countably paracompact, locally compact screenable space which is not paracompact? It is shown that the existence of such a space of cardinality $\omega_{1}$ is equivalent to the existence of a ladder system on some stationary set that is thin and countably metacompact. In addition, the existence of an example of larger cardinality is characterized by the existence of a thin, countably metacompact ladder system on a stationary subset of the same cardinal with an additional property. Section 6 is a short discussion of the question whether there may exist a thin countably metacompact ladder system on $\omega_{1}$. In the final section we establish some results connecting our ladder system properties to other known ladder system properties.

2. CH and the uniformization properties. In this section we prove that for each $n \in \omega$ it is consistent with $\mathrm{CH}$ that every ladder system satisfies $\mathcal{P}_{n+1}$ but fails to satisfy $\mathcal{M}_{n}$. Hence, all the uniformization properties are distinct, even assuming $\mathrm{CH}$. In the case $n=1$ this implies the consistency that every ladder system space $X_{L}$ is countably paracompact in a strong sense but not normal. Our proof is based on a theorem of Shelah (Theorem 9 below). A proof of Shelah's theorem has only been published for the case $n=0$. The proof of Shelah's theorem for all other $n \in \omega$ is essentially the same.

Let $\bar{L}=\left\langle L_{\alpha}: \alpha \in S\right\}$ be a ladder system on a stationary set $S$ of countable limit ordinals, and let $f \in \omega^{\omega_{1}} \omega$. We will define a notion of forcing $P=P_{f, \bar{L}}$ that adjoins a function $g: \omega_{1} \rightarrow[\omega]^{n+2}$ such that for all $\alpha \in S$,

- $g\left\lceil L_{\alpha}\right.$ is eventually constant,

- $f(\alpha) \in g(\beta)$ for all but finitely many $\beta \in L_{\alpha}$. 
Definition 2. A condition $p \in P$ satisfies

- $p: \delta+1 \rightarrow[\omega]^{n+2}$ for some $\delta<\omega_{1}$,

- if $\alpha \leq \delta$ is in $S$, then $p \nmid L_{\alpha}$ is eventually constant, say with value $F_{\alpha}$, and $p(\alpha) \in F_{\alpha}$.

Lemma 3 (Extension Lemma). Let $p: \delta+1 \rightarrow[\omega]^{n+2}$ be a condition in $P$. Given $\beta>\delta$ and $x \in[\omega]^{n+2}$, there is an extension $q$ of $p$ with $\operatorname{dom} q=$ $\beta+1$ and $q(\beta)=x$.

Proof. We prove this by induction on $\beta$ for all sets $x \in[\omega]^{n+2}$. The cases where $\beta$ is a successor ordinal or $\beta \notin S$ are trivial. If $\beta$ is a limit ordinal in $S$, we let $\left\{\beta_{m}: m<\omega\right\}$ list $L_{\beta} \backslash \delta+1$ in increasing order. We apply our induction hypothesis repeatedly to obtain a sequence $\left\{p_{m}: m<\omega\right\}$ such that

- $p_{0} \leq p$,

- $p_{m+1} \leq p_{m}$,

- $\operatorname{dom} p_{m}=\beta_{m}+1$,

- $p_{m}\left(\beta_{m}\right)=\{f(\beta), \ldots, f(\beta+n+1)\}$.

Once this is done, we define

$$
q=\bigcup_{m \in \omega} p_{m} \cup\{\langle\beta, x\rangle\} .
$$

Corollary 4. Given $p: \delta+1 \rightarrow[\omega]^{n+2}$ in $P$, a finite set $A$ of ordinals in $\omega_{1} \backslash(\delta+1)$, and a set $x \in[\omega]^{n+2}$, there is $q \leq p$ in $P$ such that $\operatorname{dom} q=$ $\max (A)+1$ and $q(\beta)=x$ for all $\beta \in A$.

Proposition 5. The set of $N \prec H(\lambda)$ countable with $\{f, \bar{L}, P\} \in N$ satisfying the following forms a club: For any $A \subseteq \delta=N \cap \omega_{1}$ of order type $\omega$ cofinal in $\delta$, and any $x \in[\omega]^{n+2}$, and given $p \in N \cap P$, there is an $(N, P)$ generic sequence $\left\{p_{n}: n \in \omega\right\}$ below $p$ such that the function defined by $\bigcup\left\{p_{n}: n \in \omega\right\}$ is eventually constant with value $x$ on $A$.

Proof. We can assume that there is a sequence $\left\langle N_{i}: i<\omega\right\rangle$ of countable elementary submodels of $H(\lambda)$ such that

- $N_{i} \in N_{i+1}$,

- $N=\bigcup_{n \in \omega} N_{i}$,

- $\{f, \bar{L}, P, p\} \in N_{0}$.

Let $\left\{D_{n}: n \in \omega\right\}$ list the dense open subsets of $P$ that are elements of $N$, and let $\delta_{i}=N_{i} \cap \omega_{1}$ for $i<\omega$. By enlarging $A$, we may assume that $\left\{\delta_{i}: i<\omega\right\} \subseteq A$ - this will not affect the order type of $A$ as the $\delta_{i}$ 's are cofinal in $\delta$. We will define $\left\{p_{n}: n \in \omega\right\}$ by induction on $n$ so that

(1) $p_{0}=p$,

(2) $p_{n+1} \leq p_{n}$, 
(3) $p_{n+1} \in D_{n}$,

(4) if $\alpha \in A \cap \operatorname{dom} p_{n+1} \backslash \operatorname{dom} p_{n}$, then $p_{n+1}(\alpha)=x$.

Given $p_{n}$, we first choose $i$ large enough that $\left\{p_{n}, D_{n}\right\} \in N_{i}$. Since $A \cap N_{i}$ is finite, we can apply Corollary 4 inside $N_{i}$ with $p_{n}$ and $A \cap N_{i}$ in place of $p$ and $A$ to obtain a condition which we shall denote by $q_{n}$. Now inside $N_{i}$, we extend $q_{n}$ to $p_{n+1} \in D_{n}$. Clearly $p_{n+1}$ has all the properties required, as does the sequence $\left\{p_{n}: n \in \omega\right\}$.

Corollary 6. $P$ is totally proper. More generally, if $N \prec H(\lambda)$ is countable with $\{\bar{L}, P, f\} \in N, p \in N \cap P$, and $x \in[\omega]^{n+2}$, then there is a totally generic $q \leq p$ such that $\operatorname{dom} q=\delta+1\left(\right.$ where $\left.\delta=N \cap \omega_{1}\right)$ and $q(\delta)=x$.

Proof. We apply Proposition 5 with $L_{\delta}$ in place of $A$, and $\{f(\delta), \ldots$, $f(\delta)+n+1\}$ in place of $x$. The sequence $\left\{p_{n}: n<\omega\right\}$ will have a lower bound $q$ with domain $\delta+1$, so we can define $q(\delta)=x$ as required.

The following corollary is the place where it is crucial that forcing conditions map into the set $[\omega]^{n+2}$ instead of into $[\omega]^{n+1}$.

COROLlary 7 . There is a simple $n+2$-completeness system $\mathbb{D}$ such that $P$ is $\mathbb{D}$-complete.

Proof. Recall that a completeness system is called simple if there is a first order formula $\psi$ such that

$$
\mathbb{D}(N, P, p)=\left\{A_{x}: x \text { a finitary relation on } N\right\},
$$

where

$$
A_{x}=\{G \in \operatorname{Gen}(N, P):\langle N \cup \mathcal{P}(N), \in\rangle \models \psi(G, x, p, P, N)\} .
$$

In our case, the formula $\psi$ says that "if $x$ is a pair $\langle y, z\rangle$ such that $y$ is an $\omega$-sequence cofinal in $N \cap \omega_{1}$ and $z \in \omega$, then $\bigcup G\lceil y$ is eventually constant and $z$ is an element of this limit value."

Note that $P$ is $\mathbb{D}$-complete, because if $x=\left\langle L_{\delta}, f(\delta)\right\rangle$, then any member of $A_{x}$ has a lower bound. Thus we need only show that $\mathbb{D}$ is an $n+2$ completeness system. To see this, let $\left\{x_{i}: i<n+2\right\}$ be a set of $n+2$ finitary relations on $N$; we must show that $\bigcap_{i<n+2} A_{x_{i}}$ is nonempty.

The nontrivial case is where all $x_{i}$ satisfy the hypothesis of the implication in the formula $\psi$. Let $x_{i}=\left\langle y_{i}, z_{i}\right\rangle$. Let $A=\bigcup_{i<n+2} y_{i}$. Then $A$ has order type $\omega$. We apply Proposition 5 to this $A$ with $\left\{z_{0}, \ldots, z_{n+1}\right\}$ in place of $x$. The sequence $\left\{q_{n}: n \in \omega\right\}$ that the conclusion gives us then generates a member of $A_{x_{i}}$ for every $i<n+2$.

Proposition 8. $P$ is $<\omega_{1}$-proper.

Proof. That $P$ is $\alpha$-proper for every $\alpha$ follows by induction on $\alpha$ using Corollary 6. 
Ensuring the failure of $\mathcal{M}_{n}$. We need the following result of Shelah (see [10, Chapter VIII, Claim 4.10] for the proof in the case $n=0$; the proof of the general case is similar).

TheOREM 9. Let $\mathbb{P}=\left\langle P_{\alpha}, \dot{Q}_{\alpha}: \alpha<\alpha_{0}\right\rangle$ be an iteration with countable support such that each $\dot{Q}_{\alpha}$ is $<\omega_{1}$-proper and $\mathbb{D}_{\alpha}$-complete for some simple $n+2$-completeness system $\mathbb{D}_{\alpha}$. Suppose that $\left\langle N_{i}: i \leq \beta\right\rangle$ is a countable increasing continuous sequence of countable models such that

- $\left\langle N_{j}: j \leq i\right\rangle \in N_{i+1}$,

- $\xi \leq \zeta \in N_{0} \cap \alpha_{0}+1$,

- $\mathbb{P} \in N_{0}$.

Suppose further that $\left\langle N_{i} \cap \alpha_{0}: i \leq \beta\right\rangle$ is long for $(\xi, \zeta),\left(G_{i}: i<n+2\right)$ are directed subsets of $P_{\xi} \cap N_{\beta}, r_{i} \in P_{\xi}$ is a lower bound for $G_{i}$ for each $i<n+2, G_{i} \cap N_{0}=G_{j} \cap N_{0}$ for all $i, j<n+2$, and for all $\eta \leq \beta$, $G_{i} \cap N_{\eta} \in \operatorname{Gen}\left(N_{\eta}, P_{\xi}\right)$. Suppose also that $p \in P_{\zeta} \cap N_{0}$ and $p\left\lceil\xi \in G_{0}\right.$. Then there is a directed $G^{*} \subseteq P_{\zeta} \cap N_{0}$ such that $G_{0} \cap N_{0} \subseteq G^{*}, G^{*} \in \operatorname{Gen}\left(N_{0}, P_{\zeta}, p\right)$, and

$$
r_{i} \Vdash_{P_{\xi}} "\left\{q\left\lceil[\xi, \zeta): q \in G^{*}\right\} \text { has a lower bound in } P_{\zeta} / P_{\xi} "\right.
$$

for all $i<n+2$.

Our iteration $\mathbb{P}$ is a countable support iteration where at even stages we force with $\left({ }^{<\omega_{1}} \omega, \supseteq\right)$ and at odd stages we force with $P_{f, \bar{L}}$ for some $f$ and $\bar{L}$. Clearly a book-keeping argument will take care that all ladders satisfy $\mathcal{P}_{n+1}$ in the extension so the crux of the matter is to prove that none of the ladder system spaces satisfy $\mathcal{M}_{n}$.

Suppose that $p \in P_{\omega_{2}}$ forces that the ladder system space built from $(\dot{S}, \dot{\bar{L}})$ is $\mathcal{M}_{n}$. We may assume that $(S, \bar{L})$ are in the ground model as we can find some limit stage $\alpha<\omega_{2}$ with $(S, \bar{L}) \in V\left[G_{\alpha}\right]$. The first thing we do is force with $\left({ }^{<\omega_{1}} \omega, \supseteq\right)$. This adjoins a function $f: \omega_{1} \rightarrow \omega$. Let $\dot{g}$ be a $P_{\omega_{2}}$-name such that

$$
p \Vdash \dot{g}: \omega_{1} \rightarrow[\omega]^{n+1} \text { uniformizes } \check{f} \text { in the sense of } \mathcal{M}_{n} .
$$

Let $\left\langle N_{i}: i \leq \beta\right\rangle$ be a sequence of models as in the assumptions of Theorem 9 with $\xi=1$ and $\zeta=\omega_{2}$, and with $\dot{g}, f$ all in $N_{0}$.

Let $\delta=N_{0} \cap \omega_{1}$, and let $G_{i}$ be chosen as in the assumptions of Theorem 9 so that $\bigcup G_{i}(\delta)=i$ for each $i<n+2$. This is easy as $\left(<\omega_{1} \omega, \supseteq\right)$ is countably complete.

Let $G^{*}$ be as in the conclusion of the theorem. From $G^{*}$, we can decode the values of $\dot{g}(\gamma)$ for all $\gamma<\delta$. Since $p \in G^{*}$, we know that $\dot{g}$ must uniformize $f$. Consider the decided sequence of values $\left(g(\gamma): \gamma \in L_{\delta}\right)$. These sets are of size $n+1$, so there are at most $n+1$ values $k$ such that $k \in g(\gamma)$ for all but finitely many $\gamma \in L_{\delta}$. This means that from $G^{*}$ we can decode 
the value of $f(\delta)$ up to a set of $n+1$ possible values. Take $i<n+2$ such that $i$ is not one of these values. This is a contradiction, as $r_{i} \Vdash f(\delta)=i$ and $r_{i}$ can be extended to a lower bound for $G^{*}$.

3. Consistency of thin ladder systems. In this section we give two proofs of the consistency of the existence of thin ladder systems. The first proof is from $\mathrm{V}=\mathrm{L}$, more specifically from Devlin's $\diamond^{\#}$ (see [5]). For our purposes we will say that a sequence $\left\{\mathcal{N}_{\alpha}: \alpha \in \omega_{1}\right\}$ is a $\diamond^{\#}$-sequence if

(1) each $\mathcal{N}_{\alpha}$ is a countable transitive model of a suitable portion of ZFC,

(2) $\left\{\mathcal{N}_{\alpha} \cap \mathcal{P}(\alpha): \alpha \in \omega_{1}\right\}$ forms a $\diamond^{+}$-sequence,

(3) $\left\{\alpha: \alpha=\left(\omega_{1}\right)^{\mathcal{N}_{\alpha}}\right\}$ is stationary.

For a more precise formulation of $\diamond^{\#}$ see [5].

THEOREM 10. $\diamond^{\#}$ implies the existence of a thin ladder system on a stationary subset of $\omega_{1}$.

REMARK. Kunen has shown that $\mathrm{V}=\mathrm{L}$ implies the existence of a thin ladder system defined on all of $\omega_{1}$.

Proof. Fix a $\diamond^{\# \text {-sequence }}\left\{\mathcal{N}_{\alpha}: \alpha \in \omega_{1}\right\}$ and let $S=\left\{\alpha: \alpha=\left(\omega_{1}\right)^{\mathcal{N}_{\alpha}}\right\}$. So $S$ is stationary. We define the ladder system on $S$. Fix $\alpha \in S$ and let $\left\{f_{k}: k \in \omega\right\}$ be an enumeration of $\mathcal{N}_{\alpha} \cap{ }^{\alpha} \omega$. Define $L_{\alpha}$ recursively: Fix $n_{0}$ minimal such that

$$
\mathcal{N}_{\alpha}=" f_{0}^{-1}\left(n_{0}\right) \text { is uncountable." }
$$

We can do this since $\mathcal{N}_{\alpha}$ "thinks" that $\alpha$ is $\omega_{1}$ and hence that $f_{0}$ is a function from $\omega_{1}$ to $\omega$. Let $I_{0}=f_{0}^{-1}\left(n_{0}\right)$. Having defined $I_{k}$ so that $\mathcal{N}_{\alpha} \models$ " $I_{k}$ is uncountable", fix $n_{k+1}$ so that

$$
\mathcal{N}_{\alpha} \models \text { " } I_{k} \cap f_{k+1}^{-1}\left(n_{k+1}\right) \text { is uncountable." }
$$

So $I_{0} \supseteq \cdots \supseteq I_{k} \supseteq \cdots$ and each $I_{k}$ is unbounded in $\alpha$. Choose an increasing cofinal $\omega$-sequence $L_{\alpha}$ in $\alpha$ such that $L_{\alpha} \subseteq^{*} I_{k}$ for each $k \in \omega$. Clearly $f_{k}\left\lceil L_{\alpha}\right.$ is eventually constant for each $k \in \omega$.

To see that $\mathcal{L}=\left\{L_{\alpha}: \alpha \in S\right\}$ is thin, fix $f: \omega_{1} \rightarrow \omega$ arbitrary. By the property of being a $\diamond^{\#}$-sequence, $C=\left\{\alpha: f\left\lceil\alpha \in \mathcal{N}_{\alpha}\right\}\right.$ is club. Clearly, by construction, $f\left\lceil L_{\alpha}\right.$ is eventually constant for any $\alpha \in S \cap C$.

Our next proof is a forcing construction of a thin ladder system. We obtain the model by collapsing a Mahlo cardinal to $\omega_{1}$. First we define a single forcing that collapses $\omega_{1}$ and preserves Mahloness of $\kappa$. Let $Q$ be the set of triples $(p, F, r)$ such that

(1) $p$ is a function from $\operatorname{dom} p \in \omega$ to $\omega_{1}$ such that $n<m<\operatorname{dom} p$ implies $p(n)<p(m)$,

(2) $F$ is a finite subset of $\omega_{1} \omega$, 
(3) $r: F \rightarrow \omega$

(4) $\left\{\alpha \in \omega_{1}: \forall f \in F(f(\alpha)=r(f))\right\}$ is uncountable.

We order $Q$ by $(p, F, r) \leq(q, G, s)$ if

(1) $p$ extends $q, F \supseteq G, r$ extends $s$,

(2) $p(n) \in g^{-1}(s(g))$ for each $n \in \operatorname{dom} p \backslash \operatorname{dom} q$ and each $g \in G$.

Since $|Q|=2^{\aleph_{1}}$ we have

LEMMA 11. $Q$ is $\left(2^{\aleph_{1}}\right)^{+}-c c$.

This cannot be improved: Given $\left\{f_{\alpha}: \alpha<2_{1}^{\aleph}\right\} \subseteq \omega^{\omega_{1}} \omega$ such that $\left\{f_{\alpha}^{-1}(0)\right.$ : $\left.\alpha<2^{\aleph_{1}}\right\}$ forms an almost disjoint family, the conditions $\left(\emptyset,\left\{f_{\alpha}\right\},\left\langle f_{\alpha}, 0\right\rangle\right)$ are pairwise incompatible (any common extension of two such conditions would violate (4)).

Let $g$ be a $Q$-name for the generic function $\bigcup\{p: \exists F, r(p, F, r) \in \Gamma\}$ and let $L$ be a $Q$-name for the range of $g$. Then $L$ is an $\omega$-sequence cofinal in $\left(\omega_{1}\right)^{\mathrm{V}}$, hence $\omega_{1}$ is collapsed by $Q$.

It is easy to verify that for each $f \in \omega^{\omega_{1}} \omega$, the set $D_{f}=\{(p, F, r) \in Q$ : $f \in F\}$ is dense in $Q$. Thus, the following lemma holds:

LEMMA 12. For any $f \in{ }^{\omega_{1}} \omega, \Vdash_{Q}$ " $\check{f}$ is eventually constant on L."

Let $\kappa$ be Mahlo. So, the set of inaccessible ordinals less than $\kappa$ is stationary in $\kappa$. Let $C=\left\{\kappa_{i}: i \in \kappa\right\}$ be an increasing enumeration of all cardinals in $\kappa$. So $C$ is club. Let $S$ be the set of inaccessibles. We fix an iteration $\left\langle P_{i}, Q_{i}: i \in \kappa\right\rangle$ as follows. For each $i \in \kappa$ let $Q_{i}$ be defined recursively as follows.

(1) If $i$ is a successor and $\Vdash_{P_{i}}$ " $\kappa_{i}$ is uncountable," let $Q_{i}$ be $\operatorname{Fn}\left(\omega, \kappa_{i}\right)$. So $Q_{i}$ collapses $\kappa_{i}$ to $\omega$.

(2) If $\kappa_{i}$ is inaccessible, let $Q_{i}$ be a $P_{i}$-name for $Q$.

(3) Otherwise let $Q_{i}$ be the trivial poset.

Let $P_{\kappa}$ be the finite support iteration of the $Q_{i}$ 's (countable also works). It easily follows that $P_{\kappa}$ has the $\kappa$-cc. It also follows that for each inaccessible $\kappa_{i}, P_{i}$ is $\kappa_{i}$-cc and collapses all $\kappa_{j}$ for $j<i$ to countable ordinals. Hence $\Vdash_{P_{i}} \kappa_{i}=\omega_{1}$. So $Q_{i}$ adds an $\omega$-sequence cofinal in $\kappa_{i}$. For each $\delta \in S$ let $L_{\delta}$ be the $P_{\delta+1}$-name for the ladder added by $Q_{\delta}$. We work with the ladder system $L=\left\{L_{\delta}: \delta \in S\right\}$.

Given any $P_{\kappa}$-name $f$ for a function $f: \kappa \rightarrow \omega$, by $\kappa$-cc the set of $\delta$ for which there is a $P_{\delta}$-name $f_{\delta}$ such that $\Vdash_{P_{\kappa}} f_{\delta}=f\lceil\delta$ is club. Thus, by the lemma above, for any $P_{\kappa}$-name $f$ for a function $\kappa \rightarrow \omega$, the set of $\delta$ for which $\Vdash_{P_{\kappa}}$ " $f\left\lceil L_{\delta}\right.$ is eventually constant" is club on $S$.

Finally, by $\kappa$-cc it follows that $S$ remains stationary in $\mathrm{V}^{P_{\kappa}}$. Thus the ladder system is thin in $\mathrm{V}^{P_{\kappa}}$. 
Hence, the existence of thin ladders is consistent. However, such ladder systems are very unstable. If $P$ is a finite support iteration of length $\omega_{1}$, then $P$ adds a function $g: \omega_{1} \rightarrow \omega$ that is $\operatorname{Fn}\left(\omega_{1}, \omega,<\omega_{1}\right)$-generic over the universe. For a ladder system $L$ in the ground model, this $g$ has the property that $g\left\lceil L_{\alpha}\right.$ has infinite range for every $\alpha \in \omega_{1}$. Thus, the ladder $L$ fails to have property $H_{1}$ in the extension. On the other hand, if $P$ is a countable support iteration of proper posets of length at least $\omega_{1}$, then $P$ adds a function $g$ that is $\operatorname{Fn}\left(\omega_{1}, \omega,<\omega_{1}\right)$-generic over the universe. This $g$ has the property that $g \nmid L_{\alpha}$ is eventually one-to-one for stationary many $\alpha$. This stationary set remains stationary by the tail of the forcing, thus, the ladder $L$ fails to have property $G_{3}$ in the extension.

Thus we have the following:

TheOREM 13. Suppose that $L$ is a ladder system and that $\mathbb{P}$ is a finite or countable support iteration of length at least $\omega_{1}$ (of proper posets). Then $\Vdash_{\mathbb{P}} L$ is not thin.

REMARK. F. Hernandez-Hernandez has constructed a proper poset that forces a thin ladder system [8]. The poset is proper but it is an $\omega_{2}$-length countable support iteration of nonproper posets.

Next we consider ladder systems on stationary subsets of cardinals $\kappa$ $>\omega_{1}$. For these cardinals, relatively weak assumptions imply that no such ladder is thin.

TheOREM 14. Let $\kappa$ be a regular cardinal. Suppose there is a cardinal $\lambda$ such that $\lambda^{\omega}<\kappa \leq 2^{\lambda}$. Then there is no thin ladder system on any stationary subset $S$ of $\{\alpha<\kappa: \operatorname{cf}(\alpha)=\omega\}$.

Proof. Let $L=\left\{L_{\alpha}: \alpha \in S\right\}$ be a ladder system. We first wish to show that there is a set $\mathcal{F}$ of less than $\kappa$ functions from $\kappa$ into $\omega$ such that every countable function from $\kappa$ into $\omega$ is extended by some function from $\mathcal{F}$. To this end, identify $\kappa$ with a subset of the space $2^{\lambda}$, and let $\mathcal{B}$ be any base for $2^{\lambda}$ of cardinality $\lambda$. Given any countable subset $A=\left\{a_{n}\right\}_{n \in \omega}$ of $\kappa$, find $B_{n} \in \mathcal{B}$ with $a_{n} \in B_{n}$ and $B_{n} \cap\left\{a_{i}: i<n\right\}=\emptyset$. Then

$$
\mathcal{P}=\left\{\kappa \cap B_{n} \backslash \bigcup_{i>n} B_{i}: n \in \omega\right\} \cup\left\{\kappa \backslash \bigcup_{i \in \omega} B_{i}\right\}
$$

is a partition of $\kappa$ each element of which contains at most one member of $A$. Since the hypothesis implies $\kappa>\mathfrak{c}$, we see that there is a set $\mathcal{F}$ of $\lambda \cdot \mathfrak{c}$, in particular less than $\kappa$, functions from $\kappa$ into $\omega$ as required.

We may fix, for each $\alpha \in S$, a function $f_{\alpha}: L_{\alpha} \rightarrow \omega$ such that the range of $f_{\alpha}$ is unbounded in $\omega$. Since $|\mathcal{F}|<\kappa$ and $\kappa$ is regular, there is an $f \in \mathcal{F}$ and a stationary $S^{\prime} \subset S$ such that $f_{\alpha}=f$ for each $\alpha \in S^{\prime}$, i.e. the coloring $f$ is unbounded on a stationary set of ladders, so $L$ is not thin. 
Corollary 15. (1) Assume the Continuum Hypothesis. Then there is no thin ladder system on any stationary subset of $\left\{\alpha<\omega_{2}: \operatorname{cf}(\alpha)\right.$ $=\omega\}$.

(2) Assume the Singular Cardinal Hypothesis. If $\kappa>\mathfrak{c}$ is regular, and not strongly inaccessible or the successor of a singular strong limit of countable cofinality, then there is no thin ladder system on any stationary subset of $\{\alpha<\kappa: \operatorname{cf}(\alpha)=\omega\}$.

Proof. For (1), CH implies that the hypothesis of the theorem is satisfied with $\kappa=\omega_{2}$ and $\lambda=\omega_{1}$. For (2), it is not difficult to show that under the Singular Cardinal Hypothesis, the hypothesis on $\kappa$ in (2) is equivalent to the hypothesis on $\kappa$ in the theorem.

4. Subspaces of $\omega_{1}^{2}$. Recently N. Kemoto and others have been systematically studying separation properties of products of ordinals and their subspaces. One of the more interesting questions left open by this investigation is the following:

QUESTION 3. Is every countably paracompact subspace of $\omega_{1}^{2}$ normal?

In [9] the following characterization was proven.

Theorem 16. For each $X \subseteq \omega_{1}^{2}, X$ is normal if and only if $X$ is countably paracompact and strongly collectionwise Hausdorff.

In addition it was shown in the same paper that $\omega_{1}^{2}$ is hereditarily collectionwise Hausdorff. Hence every normal subspace of $\omega_{1}^{2}$ is countably paracompact. Also, any countably paracompact nonnormal subspace of $\omega_{1}^{2}$ would be an example of a countably paracompact first countable collectionwise Hausdorff not strongly collectionwise Hausdorff space. In any model of ZFC where first countable countably paracompact spaces are strongly collectionwise Hausdorff, we have a positive answer to Question 3. Although it is an open question (due to P. Nyikos) whether $\mathrm{V}=\mathrm{L}$ implies that countably paracompact first countable spaces are strongly collectionwise Hausdorff, it was shown in [9] that Question 3 still has a positive answer assuming $\mathrm{V}=\mathrm{L}$.

In this section we present a general construction of a subspace of $\omega_{1}^{2}$ using a ladder system on a stationary subset of $\omega_{1}$. We consider two special instances of this construction. The first instance we consider is always nonnormal and we prove that it is countably paracompact if and only if the ladder system is thin and countably metacompact. For the second instance we find related conditions on the ladder system that characterize when the space is countably paracompact and nonnormal. Finally, we establish some relationship between the two sets of conditions.

Fix a ladder system $L$ on a stationary $S \subseteq \omega_{1}$ with the property that each $L_{\alpha}$ consists of successor ordinals. Let $B$ be a nonstationary subset of 
$\omega_{1}$ such that $B \cap S=\emptyset$. Consider the following subspace of $\omega_{1}^{2}$ :

$$
\left\{(\xi, \alpha+1): \alpha \in S, \xi \in L_{\alpha} \cup\{\alpha\}\right\} \cup\{(\beta, \gamma): \beta \in B, \gamma \in S \backslash \beta+1\} .
$$

ExAmple 1. Set $B=\bigcup\left\{L_{\alpha}: \alpha \in S\right\}$ and denote this space by $Z_{L}$. Notice that by assumption on the ladder system, for each $(\beta, \gamma) \in Z_{L}$, if $\beta \notin S$ then $\beta$ is a successor. Also notice that the closed discrete set $\{(\alpha, \alpha+1): \alpha \in S\}$ can be separated by a disjoint family of open sets, but, by stationarity of $S$, it cannot be separated by a discrete family. Hence the space is not normal.

THEOREM 17. $Z_{L}$ is countably paracompact if and only if $L$ is thin and countably metacompact.

Proof. Assume that $L$ is thin and countably metacompact. Fix a decreasing sequence $\left(D_{n}\right)_{n \in \omega}$ of closed subsets of $Z_{L}$ such that $\bigcap D_{n}=\emptyset$. Let $E_{n}=\left\{\alpha \in S:(\alpha, \alpha+1) \in D_{n}\right\}$ and define $f_{0}: S \rightarrow \omega$ by $f_{0}(\alpha)=$ $\max \left\{n: \alpha \in E_{n}\right\}$. Fix a corresponding $g_{0}: \omega_{1} \rightarrow[\omega]^{<\aleph_{0}}$ uniformizing $f_{0}$ in the appropriate sense. Let

$$
\begin{aligned}
& W_{n}^{\prime}=\left\{(\beta, \alpha+1): \beta \in L_{\alpha}, f_{0}(\alpha)=n \text { and } n \in g_{0}(\beta)\right\}, \\
& W_{n}=\left(\{(\alpha, \alpha+1): \alpha \in S\} \cap D_{n}\right) \cup \bigcup_{i \geq n} W_{i}^{\prime} .
\end{aligned}
$$

Notice that $W_{n}$ is an open neighborhood of the set $D_{n} \cap\{(\alpha, \alpha+1): \alpha \in S\}$.

To define neighborhoods at the rest of the points of $D_{n}$ first note that since $\omega_{1}$ is countably compact, for each successor $\beta$, there is an $n_{\beta}$ such that $\{\beta\} \times \omega_{1} \cap D_{n}=\emptyset$ for each $n \geq n_{\beta}$. Letting $f_{1}(\beta)=n_{\beta}$ and applying $G_{1}$, we may fix a club $C$ consisting of limit ordinals such that $f_{1}^{\prime \prime} L_{\alpha}$ is finite for each $\alpha \in C \cap S$.

For each $\gamma \in C$ let $\gamma^{+}$be the minimal element of $C$ above $\gamma$. For each $\gamma \in C$ the space $Z_{L} \cap\left(\gamma, \gamma^{+}\right]^{2}$ is a clopen metrizable subspace of $Z_{L}$. Thus, there are open sets $O_{n}^{\gamma} \supseteq D_{n} \cap\left(\gamma, \gamma^{+}\right]^{2}$ such that

$$
\bigcap_{n \in \omega} \bar{O}_{n}^{\gamma}=\emptyset \text {. }
$$

For each $(\beta, \eta) \in Z_{L} \cap D_{n}$ with $\beta \notin S$ fix an open $U_{n}(\beta, \eta)$ such that

$$
(\beta, \eta) \in U_{n}(\beta, \eta) \subseteq\{\beta\} \times(0, \eta] \cap Z_{L}
$$

so that

(a) if $(\beta, \eta) \in\left(\gamma, \gamma^{+}\right]^{2}$ for some $\gamma \in C$ then $U_{n}(\beta, \eta) \subseteq O_{n}^{\gamma} \cap\left(\gamma, \gamma^{+}\right]^{2}$,

(b) if $\beta \in\left(\gamma, \gamma^{+}\right)$for some $\gamma \in C$ and $\eta>\gamma^{+}$then $U_{n}(\beta, \eta) \subseteq\{\beta\} \times$ $\left(\gamma^{+}, \eta\right]$.

Now let $O_{n}=\bigcup\left\{U_{n}(\beta, \eta):(\beta, \eta) \in D_{n}, \beta \notin S\right\}$ and let

$$
G_{n}=W_{n} \cup O_{n} \text {. }
$$


Claim 18. $\bigcap_{n \in \omega} \bar{G}_{n}=\emptyset$.

Proof. First fix $\beta \notin S$. Fix $n$ large enough so that for every $m \geq n$ both $\{\beta\} \times \omega_{1} \cap D_{m}=\emptyset$ and $m \notin g_{0}(\beta)$. Then $\{\beta\} \times \omega_{1} \cap G_{m}=\emptyset$ for each $m \geq n$.

Next, fix $\alpha \in S$ and consider the point $(\alpha, \alpha+1)$. For each $m \neq f_{0}(\alpha)$ it is clear that $\alpha+1 \times\{\alpha+1\} \cap W_{m}^{\prime}=\emptyset$. So

$$
\left(L_{\alpha} \cup\{\alpha\}\right) \times\{\alpha+1\} \cap W_{m}=\emptyset
$$

for each $m>f_{0}(\alpha)$. Thus for $m>f_{0}(\alpha)$, if $(\alpha, \alpha+1) \in \bar{G}_{m}$ then $(\alpha, \alpha+1)$ $\in \bar{O}_{m}$. Now consider two cases:

CAsE 1: $\alpha \in C$. In this case, $f_{1}^{\prime \prime} L_{\alpha}$ is finite, so we can fix $n_{1} \in \omega$ such that $\{\beta\} \times \omega_{1} \cap D_{m}=\emptyset$ for all $\beta \in L_{\alpha}$ and all $m \geq n_{1}$. Thus

$$
\left(L_{\alpha} \times \omega_{1}\right) \cap O_{m}=\emptyset \quad \text { for each } m \geq n_{1} .
$$

CAse 2: $\alpha \notin C$. In this case, fix $\gamma \in C$ such that $\gamma<\alpha<\gamma^{+}$. Fix $n_{1}$ such that $(\alpha, \alpha+1) \notin \bar{O}_{m}^{\gamma}$ for each $m \geq n_{1}$. Thus, by choice of the open sets $U_{m}(\beta, \eta)$ for $\beta<\gamma$ and by choice of the open sets $U_{m}(\beta, \eta)$ for $\eta>\gamma^{+}$we have $(\alpha, \alpha+1) \notin \bar{O}_{m}$ for all $m \geq n_{1}$.

In either case, $(\alpha, \alpha+1) \notin \bar{O}_{m}$ for all $m \geq n_{1}$ so $Z_{L}$ is countably paracompact.

For the converse, first suppose that $L$ is not countably metacompact, and fix a partition $f: S \rightarrow \omega$ such that for any $g: \omega_{1} \rightarrow \mathcal{P}(\omega)$, if for each $\alpha \in S, f(\alpha) \in g(\beta)$ for all but finitely many $\beta \in L_{\alpha}$, then there is a $\beta$ such that $g(\beta)$ is infinite. Consider the closed sets $E_{n}=\{(\alpha, \alpha+1): f(\alpha)=n\}$. Fix open sets $W_{n} \supseteq E_{n}$. For each $\beta \notin S$ let $g(\beta)=\{n:(\beta, \alpha+1) \in$ $W_{n}$ for some $\left.\alpha \in f^{-1}(n)\right\}$. Each $W_{n}$ is open so $f(\alpha) \in g(\beta)$ for all but finitely many $\beta \in L_{\alpha}$. Thus we may fix $\beta$ such that $g(\beta)$ is infinite. Fix $\alpha_{n} \in f^{-1}(n)$ for each $n \in g(\beta)$. Let $\gamma$ be a limit of the set $\left\{\alpha_{n}: n \in g(\beta)\right\}$. Then the family $\left\{W_{n}: n \in \omega\right\}$ is not locally finite at $(\beta, \gamma)$. So if $L$ is not countably metacompact, then $Z_{L}$ is not countably paracompact.

On the other hand, assume that $L$ is not thin, and fix a function $f$ : $\omega_{1} \rightarrow \omega$ such that $T=\left\{\alpha:\left|f^{\prime \prime} L_{\alpha}\right|=\aleph_{0}\right\}$ is stationary. Let

$$
D_{n}=\left\{(\beta, \gamma) \in Z_{L}: f(\beta) \geq n \text { and } \gamma \text { is a limit }\right\} \text {. }
$$

Notice that each $D_{n}$ is closed and $\bigcap_{n \in \omega} D_{n}=\emptyset$. Suppose that for each $n$, $U_{n}$ is an open set containing $D_{n}$ such that $U_{0} \supseteq U_{1} \supseteq \cdots$. Fix a countable elementary submodel $\mathcal{M}$ such that everything relevant, e.g., $T,\left\{D_{n}: n \in \omega\right\}$, $f,\left\{U_{n}: n \in \omega\right\}$, lies in $\mathcal{M}$ which can be chosen so that $\mathcal{M} \cap \omega_{1} \in T$. Clearly, the following claim will complete the proof.

Claim 19. Let $\alpha_{0}=\mathcal{M} \cap \omega_{1}$. Then $\left(\alpha_{0}, \alpha_{0}+1\right) \in \bigcap\left\{\bar{U}_{n}: n \in \omega\right\}$.

Proof. For each $\beta \in L_{\alpha_{0}}$, let $Z_{\beta}=\{(\beta, \gamma): \gamma>\beta$ is a limit $\} \cap Z_{L}$. Notice that $Z_{\beta} \subseteq D_{f(\beta)} \subseteq U_{f(\beta)}$. By the pressing down lemma, there is a $\gamma_{\beta}$ such that $\left(\{\beta\} \times \omega_{1} \backslash \gamma_{\beta}\right) \cap Z_{L} \subseteq U_{n}$ for each $n \geq f(\beta)$. Since all objects under 
consideration are in $\mathcal{M}$ we may assume that $\gamma_{\beta} \in \mathcal{M}$ for each $\beta \in L_{\alpha_{0}}$. Now fix $n \in \omega$ and $\eta<\alpha_{0}$. Fix $\beta \in L_{\alpha_{0}}$ such that $\beta>\eta$ and $f(\beta) \geq n$. This can be done since $\alpha_{0} \in T$. Thus, $(\beta, \alpha+1) \in U_{f(\beta)} \cap\left(\eta, \alpha_{0}\right] \times\{\alpha+1\}$. Hence, $\left(\alpha_{0}, \alpha_{0}+1\right) \in \bar{U}_{n}$ for every $n \in \omega$. Thus $Z_{L}$ is not countably paracompact.

We now present a specific case of the general construction.

ExAmple 2. Let $B \subseteq\{\alpha+\omega: \alpha \in \mathrm{LIM}\}$. Expand the ladder system on $S$ to a ladder system on $S \cup B$ by letting $L_{\beta}=\{\alpha+n: n \in \omega\}$, where $\beta=\alpha+\omega \in B$. Denote this space by $Y_{L}$. To simplify notation assume that $B \subseteq S$.

Let $X_{L}$ be the associated ladder system topology on $\omega_{1} \times\{0\} \cup S \times\{1\}$ described above. While it is possible to characterize when $Y_{L}$ is countably paracompact and normal in terms of combinatorial properties of $L$ (see comments after the proof of Theorem 20), it is simpler to consider the corresponding properties of $X_{L}$ :

THEOREM 20. $Y_{L}$ is not normal if and only if $X_{L}$ satisfies

(1) The sets $S \backslash B$ and $B$ have no disjoint open neighborhoods in $X_{L}$.

$Y_{L}$ is countably paracompact if and only if it satisfies both of the following conditions:

(2) Every countable partition of $B$ can be extended to a countable family of open sets of $X_{L}$ which is locally finite at every point of $S \backslash B$.

(3) Every countable partition of $S \backslash B$ can be extended to a countable family of open sets of $X_{L}$ which is locally finite at every point of $B$.

Proof. Assume that the ladder system satisfies (1). To see that $Y_{L}$ is not normal, consider the closed subsets $H_{1}=\{(\alpha, \alpha+1): \alpha \in S \backslash B\}$ and $H_{2}=\{(\beta, \gamma): \beta \in B$ and $\gamma \in S \backslash \beta+1\}$. Suppose that $U$ is an open set containing $H_{1}$ and $V$ is an open set containing $H_{2}$. For each $\alpha \in S \backslash B$, fix $L_{\alpha}^{\prime} \subseteq^{*} L_{\alpha}$ such that $L_{\alpha}^{\prime} \times\{\alpha+1\} \subseteq U$. Let $U_{X}$ be the corresponding open set in $X_{L}$ determined by the $L_{\alpha}^{\prime}$ 's. For each $\beta \in B$ and each $\gamma \in S \backslash \beta+1$, let $\alpha_{\beta, \gamma}<\beta$ and $\delta_{\beta, \gamma}<\gamma$ be such that

$$
\left(\alpha_{\beta, \gamma}, \beta\right] \times\left(\delta_{\beta, \gamma}, \gamma\right] \cap Y_{L} \subseteq V .
$$

For each $\beta \in B$ there is $\alpha_{\beta}<\beta$ such that $\alpha_{\beta}=\alpha_{\beta, \gamma}$ for stationarily many $\gamma \in S \backslash \beta+1$. Call this stationary set $S_{\beta}$. For each $\beta \in B$ let $L_{\beta}^{\prime}=L_{\beta} \backslash \alpha_{\beta}$. Let $V_{X}$ be the corresponding neighborhood in $X_{L}$ defined by these $L_{\beta}^{\prime}$. There is a $\beta \in B$ such that the set of $\alpha \in S \backslash B$ such that $L_{\beta}^{\prime} \cap L_{\alpha}^{\prime} \neq \emptyset$ is stationary. Let $T$ denote this stationary set of $\alpha$ 's. If there were no such $\beta$, then $B$ and $S \backslash B$ would be separated in $X_{L}$. Choose $\gamma \in S_{\beta}$ a limit of $T$. Let $\alpha \in T \cap\left(\delta_{\beta, \gamma}, \gamma\right)$. Let $\xi \in L_{\alpha}^{\prime} \cap L_{\beta}^{\prime}$. Then $(\xi, \alpha+1) \in U \cap V$. So $H_{1}$ and $H_{2}$ cannot be separated. 
Conversely, assume that $B$ and $S \backslash B$ can be separated in $X_{L}$. Note that $Y_{L}=H_{1} \cup H_{2} \cup I$, where $H_{1}$ and $H_{2}$ are defined as above and $I$ is the remaining set of isolated points. Since $H_{1}$ and $H_{2}$ are closed normal subspaces of $Y_{L}$, it follows that $Y_{L}$ is normal if and only if $H_{1}$ and $H_{2}$ can be separated. Similarly to the argument above, a separation of $B$ and $S \backslash B$ leads to a separation of $H_{1}$ and $H_{2}$.

Assume (2) and (3). To see that $Y_{L}$ is countably paracompact, suppose that $\left(D_{n}: n \in \omega\right)$ is a decreasing sequence of closed subsets with empty intersection. For each $n$ let $S_{n}=\left\{\alpha \in S \backslash B:(\alpha, \alpha+1) \in D_{n}\right\}$. Let $B_{n}=$ $\left\{\beta \in B:\left\{\gamma \in S:(\beta, \gamma) \in D_{n}\right\}\right.$ is uncountable $\}$. Then both $\left(S_{n}\right)$ and $\left(B_{n}\right)$ are decreasing sequences of closed subsets of $X_{L}$ with empty intersection. By (3) we may fix an open expansion $\left(U_{n}\right)$ of $\left(S_{n}\right)$ in $X_{L}$ such that $B \cap$ $\bigcap \bar{U}_{n}=\emptyset$. Similarly, by (3) we may fix an open expansion $\left(V_{n}\right)$ of $\left(B_{n}\right)$ with $(S \backslash B) \cap \cap \bar{V}_{n}=\emptyset$. For each $\alpha \in S_{n}$ let $\alpha_{n}<\alpha$ be such that $L_{\alpha} \backslash \alpha_{n} \subseteq U_{n}$, and similarly define $\beta_{n}<\beta$ for each $\beta \in B_{n}$. Let $C$ be club in $\omega_{1}$ such that for all $\beta \in B, n \in \omega$ and $\delta>\beta$ with $\delta \in C$, if $\beta \notin B_{n}$, then the countable set $\left\{\gamma:(\beta, \gamma) \in D_{n}\right\}$ is contained in $\delta$. As in the proof that $Z_{L}$ is countably paracompact, let $O_{n}^{\gamma}$ be an open expansion of $D_{n} \cap\left(\gamma, \gamma^{+}\right]$for each $\gamma \in C$ such that the corresponding sequence of open sets $O_{n}=\bigcup\left\{O_{n}^{\gamma}: \gamma \in C\right\}$ is locally finite. For $\beta \in B_{n}$, fix $\gamma \in C$ such that $\gamma<\beta<\gamma^{+}$and let $W_{n}(\beta)=\left(\beta_{n}, \beta\right] \times\left(\gamma^{+}, \omega_{1}\right) \cap Y_{L}$. Let $S_{n}^{\prime}$ be the set of $\alpha \in S_{n}$ such that $(\alpha, \alpha+1) \notin O_{n}$, and let $W_{n}(\alpha)=\left(\alpha_{n}, \alpha\right] \times\{\alpha+1\} \cap Y_{L}$. Finally, let

$$
W_{n}=O_{n} \cup \bigcup\left\{W_{n}(\alpha): \alpha \in S_{n}^{\prime}\right\} \cup \bigcup\left\{W_{n}(\beta): \beta \in B_{n}\right\} .
$$

By the choice of the sets $O_{n}, U_{n}$ and $V_{n}$ it follows that $\bigcap \bar{W}_{n}=\emptyset$.

Conversely, suppose that (2) or (3) fails. If (2) fails, then the partition of $B$ leads naturally to a partition of $\mathrm{H}_{2}$ into a countable discrete family of closed sets that has no open expansion that is locally finite at the points of $H_{1}$ (if $B_{n}$ is one piece of the partition, let $D_{n}=\left\{(\beta, \gamma) \in Y_{L}: \beta \in B_{n}\right\}$ ). Similarly, if (3) fails, then there is a partition of $H_{1}$ that witnesses that $Y_{L}$ is not countably paracompact: If $S_{n}$ is a piece of the partition of $S \backslash B$, let $E_{n}=\left\{(\alpha, \alpha+1): \alpha \in S_{n}\right\}$.

Clearly, items (2) and (3) can be similarly characterized by appropriately uniformizing functions $f: B \rightarrow \omega$ and $g: S \backslash B \rightarrow \omega$.

We now have two sets of sufficient conditions on a ladder system that provides for the existence of a countably paracompact subspace of $\omega_{1}^{2}$ that is not normal. We have the following theorem relating these two sets of conditions:

THEOREM 21. There is a thin countably metacompact ladder system $\widetilde{L}$ if there is a ladder system $L=\left\{L_{\alpha}: \alpha \in S\right\}$ with both of the following properties. 
(4) $X_{L}$ is countably paracompact (e.g., this happens if $L$ satisfies $\mathcal{P}_{<\omega}$ ).

(5) There is an uncountable set $B \subseteq S$ which is discrete in itself if considered with the usual topology of $\omega_{1}$, such that for every open neighborhood $U$ of $B$ in $X_{L}, \overline{\text { Succ } \backslash U}^{X_{L}}$ is not stationary in $\omega_{1}$.

Moreover, if $X_{L}$ satisfies (4) and (5), then there is a ladder system $\widehat{L}$ that satisfies (1)-(3) of Theorem 20.

Proof. Let $L=\left\{L_{\alpha}: \alpha \in S\right\}$ be such that $X_{L}$ satisfies (4) and (5). Without loss of generality, assume that $\bigcup L \subseteq$ Succ. Because $B$ is discrete in itself, we can assume that $\beta<\gamma$ whenever $\alpha_{1}, \alpha_{2} \in B$ with $\alpha_{1}<\alpha_{2}$ and $\beta \in L_{\alpha_{1}}$ and $\gamma \in L_{\alpha_{2}}$. Denote the family $\left\{L_{\alpha} \cup\{\alpha\}: \alpha \in B\right\}$ by Succ (for the reason described below). Also, we may assume, without loss of generality, that $\bigcup\left\{L_{\alpha}: \alpha \in B\right\}=\bigcup L$ (since the set Succ $\backslash \bigcup\left\{L_{\alpha}: \alpha \in B\right\}$ has nonstationary closure we may thin out $S$ and the ladders to obtain this). Let $q$ be the map from $\bigcup L \cup S$ onto $\tilde{\text { Succ }} \cup(S \backslash B)$ defined by

(i) for every $\alpha \in B, q$ collapses $L_{\alpha} \cup\{\alpha\}$ into a singleton,

(ii) for every $\beta \in S \backslash B, q(\beta)=\beta$.

Then $q$ preserves order in an obvious sense so that $\tilde{\text { Succ }} \cup(S \backslash B)$ is order isomorphic to a subset of $\omega_{1}$ with Succ being a set of successor ordinals and $S \backslash B$ being a stationary set. Define a ladder system $\widetilde{L}$ on the stationary set $S \backslash B$ by the following rule. For every $\alpha \in S \backslash B$ and every $\beta \in \tilde{\text { Succ, }} \beta \in \widetilde{L}_{\alpha}$ if and only if $L_{\alpha} \cap L_{\beta} \neq \emptyset$.

Now we show that $\widetilde{L}$ is thin. Let $\left\{\tilde{S}_{\tilde{S} u c c}{ }^{n}: n \in \omega\right\}$ be a countable partition of Succ. Then $\mathcal{B}=\left\{B^{n}: n \in \omega\right\}$ is a partition of $B$ if we set $B^{n}=$ $B \cap q^{-1}\left(\tilde{\text { Succ }}^{n}\right)$. Because $X_{L}$ is countably paracompact, $\mathcal{B}$ can be expanded to a family $\mathcal{U}=\left\{U^{n} \subset X_{L}: n \in \omega\right\}$ of open subsets of $X_{L}$ which is locally finite in $X_{L}$. Denote the set

\section{$\overline{\text { Succ } \backslash \bigcup \mathcal{U}}^{X_{L}} \cap S$}

by $F$; then $F$ is not stationary by (5). Pick an $\alpha \in S \backslash(B \cup F)$. Consider the ladder $\widetilde{L}_{\alpha}$ from $\widetilde{L}$.

Because $\alpha \notin F, L_{\alpha} \subseteq^{*} \cup \mathcal{U}$. Because $\mathcal{U}$ is locally finite in $X_{L}$, there are only finitely many $k \in \omega$ such that $L_{\alpha} \cap U^{k} \neq \emptyset$. Hence if $\alpha \in S \backslash(B \cup F)$, then $\left\{k \in \omega: L_{\alpha} \cap \bigcup\left\{L_{\gamma}^{\prime}: \gamma \in B^{k}\right\} \neq \emptyset\right\}$ is a finite set. Because $\left\{q\left(L_{\gamma}\right)\right.$ : $\left.\gamma \in B^{k}\right\}=\tilde{\operatorname{Succ}}^{k}$, the set $\left\{k \in \omega: \widetilde{L}_{\alpha} \cap \tilde{\text { Succ }}^{k} \neq \emptyset\right\}$ is finite as required.

To see that $\widetilde{L}$ is countably metacompact, let $f: S \backslash B \rightarrow \omega$. We need to define the appropriate uniformizing function $F: \tilde{\text { Succ }} \rightarrow[\omega]^{<\omega}$. Since $X_{L}$ is countably paracompact, we may fix a cofinite subset $L_{\alpha}^{\prime}$ of $L_{\alpha}$ for each $\alpha \in S \backslash B$ such that the family of $U_{n}$ 's defined by

$$
U_{n}=\bigcup\left\{L_{\alpha}^{\prime}: \alpha \in f^{-1}(n)\right\}
$$


is locally finite in $X_{L}$. Thus for each $\beta \in B,\left\{n: L_{\beta} \cap U_{n}\right\}$ is finite. Let $F(q(\beta))$ be this finite set. Since Succ $=\{q(\beta): \beta \in B\}$ this defines $F$. Since $q\left(L_{\alpha}^{\prime}\right)$ is a cofinite subset of $\widetilde{L}_{\alpha}$ for each $\alpha \in S \backslash B$, it suffices to check that $f(\alpha) \in F(q(\xi))$ for all $\alpha \in S \backslash B$ and $\xi \in L_{\alpha}^{\prime}$. Fix $\alpha \in S \backslash B$ and $\xi \in L_{\alpha}^{\prime}$. Let $n=f(\alpha)$. Then by our assumption that $\bigcup\left\{L_{\beta}: \beta \in B\right\}=\bigcup L$, there is a $\beta \in B$ such that $\xi \in L_{\beta}$. Thus $L_{\beta} \cap U_{n} \neq \emptyset$, so $n \in F(q(\beta))$. Since $q(\xi)=q(\beta)$ we are done.

For the rest of the theorem, fix a ladder system $L$ on a stationary set $S$, satisfying (4) and (5). As above, we may assume that $\bigcup L \subseteq$ Succ. Let $C$ be a club that separates the points of $B$ in the sense that for each $\gamma \in C$, there is a unique element of $B$ lying in the interval $\left(\gamma, \gamma^{+}\right)$. Without loss of generality, assume that if $\beta \in B \cap\left(\gamma, \gamma^{+}\right)$then the ladder $L_{\beta}$ also lies in this interval. Without loss of generality we may assume that $S \subseteq C$, and by reordering the points of

$$
B \cup \bigcup\left\{L_{\beta}: \beta \in B\right\},
$$

we may assume that $B \subseteq\{\gamma+\omega: \gamma \in C\}$ and that $L_{\gamma+\omega}=(\gamma, \gamma+\omega)$ for each such $\gamma+\omega \in B$. Call this resulting ladder system $\widehat{L}$. Now it is easy to verify that (5) implies (1) and that (4) implies (2) and (3).

THEOREM 22. If there is a model of in in which every ladder system space is countably paracompact, then there is a ladder system space in this model which satisfies (1)-(3) of Theorem 20.

Proof. Let $B=\left\{\alpha+\omega: \alpha \in \omega_{1}\right\}$ and for each $\alpha \in$ LIM, let $L_{\alpha+\omega}=$ $(\alpha, \alpha+\omega)$. Let $\left\{L_{\alpha}: \alpha \in \operatorname{Lim}\right\}$ be any $\boldsymbol{\phi}$-sequence extending $\left\{L_{\beta}: \beta \in B\right\}$ in this model. We prove that $X_{L}$ satisfies (1)-(3). Indeed, (2) and (3) hold since $X_{L}$ is countably paracompact. To prove (1), assume towards a contradiction that $U$ and $V$ are disjoint open neighborhoods of $B$ and Lim $\backslash B$ respectively. Since $U$ is an uncountable subset of $\omega_{1}$, there is $\beta \in \operatorname{Lim}$ such that $L_{\beta} \subset U$. It follows that $\beta \subset \operatorname{Lim} \backslash B$, hence $L_{\beta}$ is contained in $V$ modulo a finite subset, contrary to the assumption that $U$ and $V$ are disjoint.

It is easy to see that $\&$ can be replaced in Theorem 22 with a much weaker principle. For example, it is enough to assume the existence of a ladder system $\left\{L_{\alpha}: \alpha \in S\right\}$ such that for every uncountable $A \subset$ Succ there is $\alpha \in S$ with $A \cap L_{\alpha}$ infinite. Also, it is enough to assume that the ladder system constructed in the proof is countably paracompact. For a further discussion of similar problems, see Section 7 below.

5. Screenable countably paracompact spaces. A space is screenable if every open cover has a $\sigma$-disjoint open refinement. Z. Balogh [1] 
showed that normal locally compact screenable spaces are paracompact (in ZFC). But the question whether or not the same is true with "normal" replaced by "countably paracompact" remains open. P. Daniels showed that it holds under $\mathrm{V}=\mathrm{L}$ [4] or under $\mathrm{MA}_{\omega_{1}}+$ Axiom $\mathrm{R}$ [3]. In the result below, we obtain an equivalence of the problem in terms of ladder systems. Note that Daniels's results follow from this equivalence, together with Theorem 24 and Corollary 27 in the next section.

THEOREM 23. The following are equivalent:

(1) There is a countably paracompact, locally compact screenable space which is not paracompact.

(2) There is an uncountably regular cardinal $\kappa$ and a thin, countably metacompact ladder system $L$ on a stationary subset $S$ of $\{\alpha<\kappa$ : $\operatorname{cf}(\alpha)=\omega\}$ such that the ladder space restricted to any $\alpha<\kappa$ is $C W H$.

Proof. $(2) \Rightarrow(1)$. Let $\left\{L_{\alpha}: \alpha \in S\right\}$ be a ladder system satisfying the conditions of (2). The set for our space $X$ is $(\kappa \times 2) \cup E$, where $E=\{\{\alpha, \beta\}$ : $\beta \in L_{\alpha}$ or $\left.\alpha \in L_{\beta}\right\}$. The set $E$ is a set of isolated points. Let $F$ be a finite set. A neighborhood of a point of the form $(\beta, 0)$ is

$$
N(\beta, 0, F)=\{(\beta, 0)\} \cup\left\{\{\beta, \alpha\} \in E: \beta \in L_{\alpha}\right\} \backslash F
$$

and a neighborhood of $(\alpha, 1)$ is

$$
N(\alpha, 1, F)=\{(\alpha, 1)\} \cup\left\{\{\beta, \alpha\} \in E: \beta \in L_{\alpha}\right\} \backslash F
$$

Note the following:

- $\kappa \times 2$ is a closed discrete set in $X$.

- $N(\alpha, e, F)$ is the one-point compactification of a subset of $E$.

- For fixed $e<2$, the sets $N(\alpha, e, \emptyset), \alpha<\kappa$, are pairwise disjoint.

- $N(\beta, 0, \emptyset) \cap N(\alpha, 1, \emptyset)$ equals $\{\alpha, \beta\}$ if $\beta \in L_{\alpha}$, and is empty otherwise.

It follows that $X$ is locally compact, screenable, and 2-boundedly metacompact. It is not collectionwise Hausdorff, so not paracompact, by the pressing down lemma.

Note that we have not yet used any of the special properties of the ladder system. We will use them in proving that $X$ is countably paracompact. Let $c: \kappa \times 2 \rightarrow \omega$ code a countable partition of $\kappa \times 2$. It suffices to show that there is a locally finite expansion.

Let $C$ be a club witnessing thinness of the ladder system with respect to the coloring $c_{0}(\alpha)=c(\alpha, 0)$. Let $F_{\alpha} \in\left[L_{\alpha}\right]^{<\omega}$ witness countable metacompactness of the ladder system for the partition $c_{1}(\alpha)=c(\alpha, 1)$; i.e., if $\beta \in \kappa$, then the set $\left\{c_{1}(\alpha): \beta \in L_{\alpha} \backslash F_{\alpha}\right\}$ is finite. By the CWH property, we may assume that for $\alpha, \alpha^{\prime} \notin C, L_{\alpha} \backslash F_{\alpha} \cap L_{\alpha^{\prime}} \backslash F_{\alpha^{\prime}}=\emptyset$. 
Now let $F_{\beta 0}=\left\{\{\beta, \alpha\}: \beta \in L_{\alpha} \backslash F_{\alpha}, \alpha \notin C\right\}$, and $F_{\alpha 1}=\{\{\beta, \alpha\}$ : $\left.\beta \in F_{\alpha}\right\}$. Note that these are finite sets. Let $U_{n}=\bigcup_{c(\alpha, 1)=n} N\left(\alpha, 1, F_{\alpha 1}\right)$ and $V_{n}=\bigcup_{c(\beta, 0)=n} N\left(\beta, 0, F_{\beta 0}\right)$.

It remains to prove that $\left\{U_{n}: n \in \omega\right\}$ and $\left\{V_{n}: n \in \omega\right\}$ are locally finite. Any limit point of $\left\{V_{n}: n \in \omega\right\}$ would have the form $(\alpha, 1)$. Suppose $N\left(\alpha, 1, F_{\alpha 1}\right) \cap V_{n} \neq \emptyset$. Then there is $\beta$ with $c(\beta, 0)=n$ and $N\left(\alpha, 1, F_{\alpha 1}\right) \cap$ $N\left(\beta, 0, F_{\beta 0}\right) \neq \emptyset$. Thus $\beta \in L_{\alpha} \backslash F_{\alpha}$ and $\alpha \in C$. But for $\alpha \in C$, the set $\left\{c(\beta, 0): \beta \in L_{\alpha}\right\}$ is finite. It follows that $N\left(\alpha, 1, F_{\alpha 1}\right)$ meets only finitely many $V_{n}$ 's.

Finally, let us see that $\left\{U_{n}: n \in \omega\right\}$ is locally finite. If not, there would be a limit point of the form $(\beta, 0)$. If $N(\beta, 0, \emptyset) \cap U_{n} \neq \emptyset$, then there is $\alpha \in S$ with $N(\beta, 0, \emptyset) \cap N\left(\alpha, 1, F_{\alpha 1}\right) \neq \emptyset$ and $c(\alpha, 1)=n$. Then $\beta \in L_{\alpha} \backslash F_{\alpha}$ and since the $F_{\alpha}$ 's witness countable metacompactness of the ladder space, the set of possible "colors" $n=c(\alpha, 1)$ is finite. So, $N(\beta, 0, \emptyset)$ meets only finitely many $U_{n}$ 's.

$(1) \Rightarrow(2)$. Assume that there is a countably paracompact, locally compact, screenable space which is not paracompact. We will be using the following results of P. Daniels [3]. Let $Z(\kappa)$ denote the space $\kappa \cup[\kappa]^{2}$, where $[\kappa]^{2}$ is the set of isolated points, and a neighborhood of $\alpha \in \kappa$ has the form

$$
N(\alpha, F)=\{\alpha\} \cup\left\{\{\alpha, \beta\} \in[\kappa]^{2}: \beta \in \kappa \backslash F\right\},
$$

where $F$ is finite. Note that $\kappa$ is a closed discrete set of points in $Z(\kappa)$.

(i) If there is a space as in (1), then there is a countably paracompact subspace $X$ of $Z(\kappa)$ for some uncountable regular cardinal $\kappa$ such that $X$ contains $\kappa$ and $\kappa$ is unseparated in $X$, but every initial segment of $\kappa$ is separated in $X$.

(ii) Let $A_{\alpha}=\{\beta:\{\alpha, \beta\} \in X\}$ and $\Gamma=\left\{\alpha<\kappa: \exists \beta \geq \alpha\left(\left|A_{\beta} \cap \alpha\right|\right.\right.$ $\geq \omega)\}$. Then $\Gamma$ is stationary.

(iii) For each $\alpha \in \Gamma$, choose $\theta(\alpha) \geq \alpha$ such that $A_{\theta(\alpha)} \cap \alpha$ is infinite. Then there is a stationary $\Delta \subset \Gamma$ such that $\theta\lceil\Delta$ is one-to-one. Further, the set $\Omega=\left\{\alpha \in \Delta: \operatorname{cf}(\alpha)=\omega\right.$ and $\left.\sup \left(A_{\theta(\alpha)} \cap \alpha\right)=\alpha\right\}$ is also stationary.

Let $\Omega$ be as in (iii) above. By passing to the intersection of $\Omega$ with a club if necessary, we may assume that $\alpha<\beta \in \Omega$ implies $\theta(\alpha)<\beta$. For each $\alpha \in \Omega$, let $L_{\alpha}$ be a subset of $A_{\theta(\alpha)} \cap \alpha$ which is cofinal in $\alpha$ and has order type $\omega$. We claim that $L=\left\{L_{\alpha}: \alpha \in \omega\right\}$ is a ladder system having the desired properties.

That the ladder space of $L$ restricted to any $\alpha<\kappa$ is CWH follows easily from the fact (see (i) above) that $X$ is $<\kappa-\mathrm{CWH}$.

Claim 1. L is thin. 
To see this, suppose $g: \kappa \rightarrow \omega$. Since $X$ is countably paracompact, for each $\alpha$ we can find $F_{\alpha} \in[\kappa]^{<\omega}$ such that if $U_{n}=\bigcup_{g(\alpha)=n} N\left(\alpha, F_{\alpha}\right)$, then $\left\{U_{n}\right\}_{n \in \omega}$ is locally finite, and further, local finiteness at $\alpha$ is witnessed by $N\left(\alpha, F_{\alpha}\right)$. Suppose $g$ has infinite range on $L_{\alpha}$ for all $\alpha$ in a stationary set $T$. Find $\alpha \in T$ such that $F_{\beta} \subset \alpha$ for each $\beta<\alpha$. Then for all sufficiently large $\beta \in L_{\alpha}$, the point $\{\beta, \theta(\alpha)\}$ is in $N\left(\beta, F_{\beta}\right) \cap N\left(\theta(\alpha), F_{\theta(\alpha)}\right)$. It follows that $N\left(\theta(\alpha), F_{\theta(\alpha)}\right)$ meets $U_{n}$ for infinitely many $n$, a contradiction.

It remains to prove:

Claim 2. L is countably metacompact.

Let $h: \Omega \rightarrow \omega$. We need to find finite sets $H_{\alpha}, \alpha \in \Omega$, such that for each $\beta$, the set $\left\{h(\alpha): \beta \in L_{\alpha} \backslash H_{\alpha}\right\}$ is finite.

To this end, first extend $h$ to $g: \kappa \rightarrow \omega$ so that $g(\theta(\alpha))=h(\alpha)$. Apply countable paracompactness of $X$ to obtain finite sets $F_{\alpha}, \alpha \in \kappa$, with the same properties as in Claim 1. We claim that $H_{\alpha}=F_{\alpha} \cup F_{\theta}(\alpha)$ works. Suppose on the contrary that $\beta$ is such that $\left\{h(\alpha): \beta \in L_{\alpha} \backslash H_{\alpha}\right\}$ is infinite. Choose $k_{0} \in \omega$ such that $g\left(F_{\beta}\right) \subset k_{0}$. Then find $k_{0}<k_{1}<\cdots$ such that $\beta \in L_{\alpha_{n}} \backslash H_{\alpha_{n}}$ for some $\alpha_{n} \in \Omega$ with $g\left(\theta\left(\alpha_{n}\right)\right)=h\left(\alpha_{n}\right)=k_{n}$. Then the point $\left\{\beta, \theta\left(\alpha_{n}\right)\right\}$ is in $N\left(\beta, F_{\beta}\right) \cap N\left(\theta\left(\alpha_{n}\right), F_{\theta\left(\alpha_{n}\right)}\right)$ for all $n$, whence $N\left(\beta, F_{\beta}\right)$ meets $U_{k_{n}}$ for all $n$, a contradiction.

6. Consistency of thin countably metacompact ladders. In the last two sections we have shown two different topological statements to be equivalent to the existence of a ladder system that is both thin and countably metacompact. We conjecture that there may be such a ladder system.

We know various models in which there are no such ladder systems. For example, we have already seen that uncountable finite-support and countable-support iterations, and uniformizability of ladder systems (and hence $\left.\mathrm{MA}_{\omega_{1}}\right)$ kill thin ladder systems. We have also seen that $\diamond^{\#}$ implies the existence of a thin ladder system. However, a weaker diamond principle, $\diamond(S)$, kills the conjunction thin + countably metacompact on $S$.

THEOREM 24. Let $S$ be a stationary subset of the ordinals of countable cofinality in the regular uncountable cardinal $\kappa$. Then $\diamond(S)$ implies there is no thin countably metacompact ladder system on $S$.

Proof. $\diamond(S)$ implies that there are $F_{\alpha}: \alpha \rightarrow[\omega]^{<\omega}$ for $\alpha \in S$ such that, given any $F: \kappa \rightarrow[\omega]^{<\omega}$, there are stationarily many $\alpha \in S$ with $F\left\lceil\alpha=F_{\alpha}\right.$.

Suppose $L$ is a thin, countably metacompact ladder system on $S$. For each $\alpha \in S$, choose, if possible, $f(\alpha) \in \omega$ such that $f(\alpha) \notin F_{\alpha}(\beta)$ for cofinally many $\beta \in L_{\alpha}$; otherwise, let $f(\alpha)=0$. By countable metacompactness, there is $F: \kappa \rightarrow[\omega]^{<\omega}$ such that for each $\alpha \in S, f(\alpha) \in F(\beta)$ for all but finitely 
many $\beta \in L_{\alpha}$. By thinness, $F$ is eventually constant on a club $C$ of ladders. Considering $\alpha \in C \cap S$ such that $F\left\lceil\alpha=F_{\alpha}\right.$ yields a contradiction.

REMARK. It is a corollary to the above proof that a thin countably metacompact ladder also satisfies $\mathcal{P}_{<\omega}$ and that $\diamond(S)$ implies that no ladder on $S$ satisfies $\mathcal{P}_{<\omega}$.

As far as higher cardinal versions are concerned, we do not know if it is consistent for there to be any thin ladder system on any stationary subset of a regular cardinal greater than $\omega_{1}$. Under Fleissner's Axiom R [7], there is no need to consider cardinals higher than $\omega_{1}$ for the topological application of Section 5 (see Theorem 23).

Theorem $25([7])$. (Axiom $\mathrm{R})$ If $X$ is $\omega_{1}$-cwH, has local density $\leq \omega_{1}$, and has countable tightness, then $X$ is $C W H$.

Corollary 26. (Axiom $\mathrm{R}$ ) Let $\kappa>\omega_{1}$ be regular. Then there is no ladder system on a stationary subset of $\kappa$ such that the ladder space restricted to any $\alpha<\kappa$ is $C W H$.

Proof. A ladder space with the stated properties easily satisfies the hypotheses of Fleissner's theorem but not the conclusion.

COROLlary 27. ( $\mathrm{MA}_{\omega_{1}}+$ Axiom $\left.\mathrm{R}\right)$ There is no ladder system satisfying the conditions of Theorem 23(2).

Finally, we mention that we also do not know if thin $+\mathcal{M}_{0}$ is consistent, i.e., if there could be a thin, normal ladder system.

7. Relation to other ladder system properties. We now investigate the relationship between $\mathbf{0}$-type properties of ladder systems with the properties studied in the previous sections.

It is easy to see that if a ladder system $\left\{L_{\alpha}: \alpha \in \omega_{1}\right\}$ is a $\boldsymbol{\beta}_{\text {-sequence, }}$ then it satisfies $H_{1}$. To see this, suppose that $f: \omega_{1} \rightarrow \omega$ is given. Let $n$ be such that $A=f^{-1}(n)$ is uncountable. Then if the set of $\alpha$ such that $L_{\alpha} \subseteq^{*} A$ is stationary, then we see that $H_{1}$ is satisfied. Similarly one can see that the weak $\boldsymbol{\beta}$-principle that requires for each uncountable $A$ that only $L_{\alpha} \cap A$ is infinite for some $\alpha$ (hence for stationarily many $\alpha$ ), gives the property $H_{2}$.

On the other hand, if $\left\{L_{\alpha}: \alpha \in \omega_{1}\right\}$ has any one of our anti-uniformization properties, then so does the sequence $\left\{L_{\alpha}^{\prime}: \alpha \in \omega_{1}\right\}$, where

$$
L_{\alpha}^{\prime}=\left\{\beta+1: \beta \in L_{\alpha}\right\} .
$$

Each element of this sequence is disjoint from the set of limit ordinals, hence fails even the weak $\mathbf{s}$-principle.

It is possible to modify our proofs of the existence of a thin ladder system on $\omega_{1}$ to make the resulting ladder system a $\mathbf{s}$-sequence. On the other hand, 
it is consistent that there is a $\mathbf{p}$-sequence that is not $G_{3}$ (both proofs are in $[8])$.

Question 4. May there be a $\mathbf{s}$-sequence that is not $G_{3}$ ?

Concerning the uniformization properties, we have the following result:

Proposition 28. Any $\mathbf{\beta}$-sequence fails property $\mathcal{P}_{0}$.

Proof. Let $\left\{L_{\alpha}: \alpha \in S\right\}$ be a $\mathbf{s}$-sequence. Fix $f: S \rightarrow \omega$ such that $f^{-1}(0)$ is uncountable and nonstationary. Suppose that $F$ uniformizes $f$ as given by $\mathcal{P}_{0}$. Then $F^{-1}(0)$ is uncountable. Hence the set of $\alpha$ such that $L_{\alpha} \subseteq^{*} F^{-1}(0)$ is stationary. Thus there is $\alpha$ such that $f(\alpha) \neq 0$ but $F$ is eventually constant with value 0 on $L_{\alpha}$; a contradiction.

QUESTION 5. May there be a $\mathbf{s}$-sequence that satisfies $\mathcal{P}_{1}$ ? or even just $\mathcal{M}_{<\omega}$ ?

Recall that Theorem 22 of Section 4 raised the following question to which a positive solution would give an example of a countably paracompact subspace of $\omega_{1}^{2}$ that is not normal.

QUESTION 6. Is it consistent that there exists a weak $\mathbf{9}$-sequence, and every ladder system satisfies $\mathcal{P}_{<\omega}$ ?

In $[12]$ the following $\mathbf{p}$-principles are introduced.

Definition 29. $\mathbf{d}_{\mathrm{NS}}$ is the statement: there is a ladder system $L=$ $\left\{L_{\alpha}: \alpha \in \omega_{1}\right\}$ such that

(1) for each club $C \subseteq \omega_{1}$, the set of $\alpha$ such that $L_{\alpha} \subseteq{ }^{*} C$ contains a club,

(2) for each $A \subseteq \omega_{1}$ there is a club $C$ such that for each $\alpha \in C$ either $L_{\alpha} \subseteq^{*} A$ or $L_{\alpha} \cap A$ is finite.

LEMMA 30. If $L$ satisfies $\mathbf{s}_{\mathrm{NS}}$, then for every stationary $S \subseteq \omega_{1}$ and every partition $\left\{A_{n}: n \in \omega\right\}$ of $\omega_{1}$,

$$
\left\{\alpha \in S:\left(\exists n\left(L_{\alpha} \subseteq^{*} A_{n}\right)\right) \vee\left(\forall n\left(\left|L_{\alpha} \cap A_{n}\right|<\aleph_{0}\right)\right\}\right.
$$

is stationary.

Proof. Apply 29(2) to each $A_{n}$ and let $S^{\prime}=S \cap \bigcap_{n} C_{n}$.

Corollary 31. If $L$ is a $\mathbf{m}_{\mathrm{NS}}$-sequence, and $L$ is not thin on any stationary set, then for every stationary set there is a partition $\left\{A_{n}: n \in \omega\right\}$ of $\omega_{1}$ such that

$$
\left\{\alpha \in S: \forall n\left(\left|A_{n} \cap L_{\alpha}\right|<\aleph_{0}\right)\right\}
$$

is stationary. 
For the next definition we need some notation. Let $T$ be stationary and $h:\left[\omega_{1}\right]^{<\omega} \rightarrow P\left(\omega_{1}\right)$, and set

$$
\begin{aligned}
\mathcal{F}_{T, L} & =\left\{A \subseteq \omega_{1}:\left\{\alpha: L_{\alpha} \subseteq^{*} A\right\} \text { is club on } T\right\}, \\
Z_{h, L} & =\left\{\alpha: \exists \beta \in L_{\alpha} \forall \eta \in L_{\alpha} \backslash \beta\left(\eta \in h\left(L_{\alpha} \cap \eta\right)\right)\right\} .
\end{aligned}
$$

Definition 32 . $\boldsymbol{\beta}_{\mathrm{NS}}^{+}$is the statement: there is a ladder system $L=$ $\left\{L_{\alpha}: \alpha \in \omega_{1}\right\}$ satisfying $\boldsymbol{\beta}_{\mathrm{NS}}$ and such that, for all $X \in\left[P\left(\omega_{1}\right)\right]^{\omega_{1}}$ and all stationary $S$, there is a stationary $T \subseteq S$ and an ultrafilter $u$ on $\omega_{1}$ such that

(1) $\mathcal{F}_{T, L} \cap X=u \cap X$,

(2) for all $h:\left[\omega_{1}\right]^{<\omega} \rightarrow X \cap u, T \backslash Z_{h, L}$ is nonstationary.

Theorem 33. If $L$ satisfies $\boldsymbol{p}_{\mathrm{NS}}^{+}$and is not thin, then $\mathfrak{d}=\omega_{1}$.

Proof. Fix a partition $\left\{A_{n}: n \in \omega\right\}$ of $\omega_{1}$ such that

$$
S=\left\{\alpha: \forall n\left(\left|L_{\alpha} \cap A_{n}\right|<\aleph_{0}\right)\right\}
$$

is stationary.

Let $B_{n}=\bigcup_{m>n} A_{n}$ and let $X \subseteq P\left(\omega_{1}\right)$ be any family of sets such that $B_{n} \in X$ for all $n$. Let $T \subseteq S$ be stationary and $u$ be an ultrafilter given by the definition of $\boldsymbol{\beta}_{\mathrm{NS}}^{+}$. Note that since $T \subseteq S$, we have $B_{n} \in \mathcal{F}_{T, L} \cap X$ for each $n$. So $B_{n} \in u \cap X$ for each $n \in \omega$.

For each $\alpha \in \omega_{1}$, let $\left\{\alpha_{n}: n \in \omega\right\}$ be the increasing enumeration of $L_{\alpha}$ and define $g_{\alpha}: \omega \rightarrow \omega$ by

$$
g_{\alpha}(n)=\min \left\{m: B_{m} \cap\left\{\alpha_{i}: i \leq n\right\}=\emptyset\right\} .
$$

We claim that the family of $g_{\alpha}$ is dominating. Fix $f: \omega \rightarrow \omega$. Define $h$ : $[\omega]^{<\omega} \rightarrow u \cap X$ by $h(a)=B_{f}(|a|)$. By definition of $\boldsymbol{\alpha}_{\mathrm{NS}}^{+}$, we may fix $\alpha \in$ $Z_{h, L} \cap T$. By definition of $Z_{h, L}$ we may fix $\beta \in L_{\alpha}$ such that $\eta \in h\left(L_{\alpha} \cap \eta\right)$ for all $\eta \in L_{\alpha} \backslash \beta$. Fix $n_{0}$ such that $\alpha\left(n_{0}\right)=\beta$ and fix $n>n_{0}$. Then

$$
\alpha(n) \in h\left(L_{\alpha} \cap \alpha(n)\right)=B_{f\left(\left|L_{\alpha} \cap \alpha(n)\right|\right)}=B_{f(n)} .
$$

Since $\alpha(n) \notin B_{g_{\alpha}(n)}$ it follows that $g_{\alpha}(n)>f(n)$. Thus $f<^{*} g_{\alpha}$ and hence $\left\{g_{\alpha}: \alpha<\omega_{1}\right\}$ is dominating.

In $\left[12\right.$, Chapter 8], the consistency of $\boldsymbol{\alpha}_{\mathrm{NS}}^{+}$with saturation of the nonstationary ideal in a variant of a $P_{\max }$ extension is obtained. The continuum is $\aleph_{2}$ in this model. Unfortunately, $\mathfrak{d}=\omega_{1}$ also holds in this model [13]. Nonetheless, we conjecture that consistency of a thin ladder system satisfying weak uniformization properties should be obtainable using some $P_{\max }$ variation. Indeed, the statement that there is a stationary set carrying a thin ladder system is the negation of a $\Pi_{2}$ sentence, and the sentence asserting that every ladder system is countably metacompact is $\Pi_{2}$. 


\section{References}

[1] Z. Balogh, Paracompactness in locally Lindelöf spaces, Canad. J. Math. 38 (1986), 719-727.

[2] D. K. Burke and Z. Balogh, A total ladder system space by ccc forcing, Topology Appl. 44 (1992), 37-44.

[3] P. Daniels, When countably paracompact, locally compact, screenable spaces are paracompact, ibid. 26 (1987), 271-279.

[4] -, On collectionwise Hausdorffness in countably paracompact, locally compact spaces, ibid. 28 (1988), 113-125.

[5] K. Devlin, The combinatorial principle $\diamond^{\sharp}$, J. Symbolic Logic 47 (1982), 888-899.

[6] K. Devlin and S. Shelah, A weak version of $\diamond$ which follows from $2^{\aleph_{0}}<2^{\aleph_{1}}$, Israel J. Math. 29 (1978), 239-247.

[7] W. G. Fleissner, Left separated spaces with point-countable bases, Trans. Amer. Math. Soc. 294 (1986), 665-677.

[8] F. Hernandez-Hernandez, preprint.

[9] N. Kemoto, K. D. Smith and P. J. Szeptycki, Countable paracompactness versus normality in $\omega_{1}^{2}$, Topology Appl. 104 (2000), 141-154.

[10] S. Shelah, Proper and Improper Forcing, Springer, 1998.

[11] - A consistent counterexample in the theory of collectionwise Hausdorff spaces, Israel J. Math. 65 (1989), 219-224.

[12] W. H. Woodin, The Axiom of Determinacy, Forcing Axioms, and the Nonstationary Ideal, de Gruyter Ser. Logic Appl., de Gruyter, 1999.

[13] - private communication.

Department of Mathematics

University of Northern Iowa

Cedar Falls, IA 50614, U.S.A.

E-mail: eisworth@uni.edu

Department of Mathematics

Towson University

8000 York Road

Towson, MD 21252, U.S.A.

E-mail: paramount@yahoo.com
Department of Mathematics Auburn University 221 Parker Hall Auburn, AL 36849, U.S.A. E-mail: gruengf@mail.auburn.edu

Atkinson Faculty York University Toronto, ON M3J 1P3, Canada

E-mail: szeptyck@yorku.ca

Received 15 February 2003;

in revised form 29 October 2003 